\title{
Rickettsial diseases: the typhus group of fevers-a review
}

\author{
George Cowan
}

Epidemic louse borne typhus has historically caused massive mortality in the wake of war, famine, and great migrations. ${ }^{1}$ In the four years from 1918 in Eastern Europe and Russia there were up to 30 million cases, and three million deaths. In the recent past in Burundi typhus has infected prison inmates before spreading to the wider community. ${ }^{2}$ It remains a risk among refugee populations in all parts of the world, despite its omission from a recent review of health care in refugee camps. ${ }^{3}$

Tick borne typhus is a significant risk to human health, especially in the Eastern United States, Brazil, the Mediterranean basin, the African veld, India, and Australia. Endemic flea borne typhus occurs sporadically wherever rats and man live closely together. Scrub typhus is a hazard in many parts of South East Asia and beyond, and is the first of the rickettsial infections to show evidence of resistance to standard antibiotics. ${ }^{4}$ No useful vaccines are currently available for any of the rickettsial infections.

\section{Pathogenesis}

The rickettsiaceae are a family of obligate intracellular small Gram negative coccobacilli which infect humans chiefly through insect vectors, mostly from animal hosts, but sometimes by transovarial transmission in the insects themselves. ${ }^{5}$

The genus Rickettsia is divided into:

(1) $R$ prowazekii, the agent of classical epidemic typhus, transmitted by the human body (clothing) louse, Pediculus humanus (but not by head lice) from active human cases or from healthy carriers or subclinical cases, so-called Brill-Zinsser disease. ${ }^{6}$ Typical circumstances were evident in the Burundi outbreak, ${ }^{2}$ which started in a prison at N'Gozi in 1995 and spread to the malnourished inhabitants of refugee camps in the central highlands (over $1500 \mathrm{~m}$ ), causing over 50000 cases with a mortality of $2.6 \%$. The infectious agent in the faeces of the body louse is usually inoculated by scratching of the site of the louse bite, but in epidemics in closed communities an aerosol of dried louse faeces may be inhaled. The genome of the organism has recently been sequenced, providing new evidence of an evolutionary relationship between rickettsiae and intracellular mitochondria in general. ${ }^{7}$

(2) $R$ mooseri ( $R$ typhi), the causal agent of endemic typhus, is carried by the rat flea Xenopsylla cheopis, and typically infects man in markets, grain stores, breweries, and garbage depots. It is often a mild illness, but can become more aggressive in refugee camps, and a fatal case in the UK was infected in Spain. ${ }^{8}$
(3) The "spotted fever" group of rickettsiae, which contains a large number of species transmitted from rodents, dogs, and wild animals by ticks:

(A) $R$ rickettsii, the agent of Rocky Mountain spotted fever, so-called because of the area of its discovery, but now mainly occurring in the eastern Atlantic states of USA, especially in trekkers and hunters exposed to wild animal ticks, ${ }^{9}$ and with the potential for a severe haemorrhagic illness.

(B) $R$ conorii, the cause of tick typhus in the Mediterranean area and in India, which is transmitted by the brown dog tick Rhipicephalus sanguineus - the tick is brown, the dog not necessarily so. A recent human case in Lille in northern France was caused by a tick from a dog imported from Marseille. ${ }^{10}$

(C) $R$ africae, which is found in the African veld, is transmitted in game park areas by ticks living on cattle, hippo, and rhino. ${ }^{11}$

(D) $R$ japonica, $R$ australis, and a variety of other similar organisms, which are widely distributed in Asia and Australia, ${ }^{12}{ }^{13}$ and infect man through various species of animal ticks.

(4) $R$ tsutsugamushi, recently renamed as a new genus with only one species, Orientia tsutsugamushi, ${ }^{14}$ the agent of scrub typhus, acquired from the bite of larval trombiculid mites living on the waist high Imperata grass growing in previously cleared jungle around villages and in plantations. The area of risk includes South East Asia, the Indian subcontinent, Sri Lanka, and other Indian Ocean islands, Papua New Guinea, and North Queensland.

The rickettsial fevers are acute bacteraemic illnesses characterised by headache, mental confusion (and, in severe cases, meningoencephalitis), a macular rash, mainly on the trunk, with, in some types, a small black scab or eschar at the site of the insect bite, with local or general lymphadenopathy.

Rickettsial proliferation on the endothelium of small blood vessels releases cytokines which damage endothelial integrity, with consequent

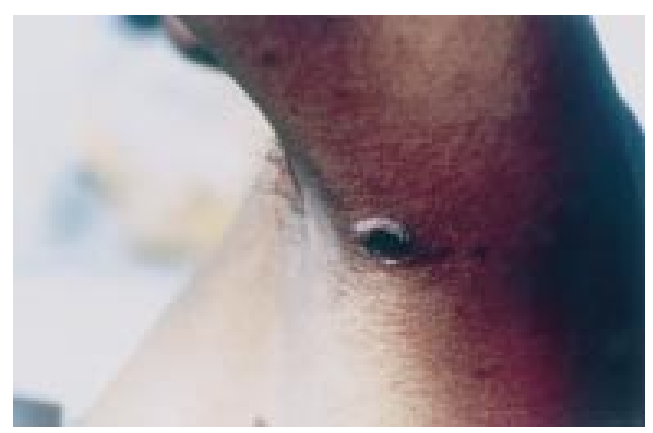

Figure 1 Scrub typhus: axillary eschar. 


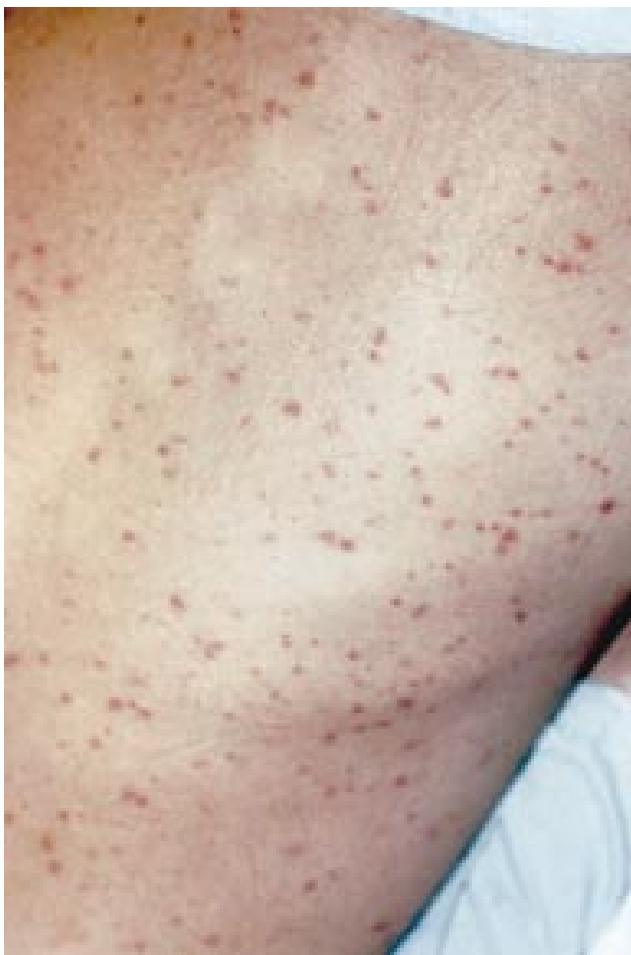

Figure 2 Classical typhus rash on trunk.

fluid leakage, platelet aggregation, and polymorph and monocyte proliferation, leading to focal occlusive endangiitis, causing microinfarctions, as in the typical "typhus nodule" of Wolhbach. ${ }^{15}$ This process especially affects the brain, cardiac and skeletal muscle, the skin, lungs and kidneys, and may cause venous thrombosis and peripheral gangrene.

\section{Clinical presentations}

The incubation period of all rickettsial infections is 12 to 15 days on average, but can extend up to 28 days.

Murine and milder cases of scrub typhus often present as non-specific fevers for a few days' duration, ${ }^{16}$ with a minimal macular rash, or no rash at all, and no eschar at the site of entry. Generalised lymphadenopathy was a characteristic feature of the milder cases seen in US soldiers in Vietnam, where tetracycline was freely prescribed for cases of "pyrexia of unknown origin" and the specific diagnosis was made serologically in retrospect.

Rocky Mountain spotted fever is a potentially serious febrile illness with a haemorrhagic rash and the possibility of diffuse intravascular coagulation and acute renal failure. Perhaps surprisingly there is no eschar at the site of the tick bite, although this is seen with the same organism in Brazil. Tick typhus in the Old World, with its many species types, varies in severity but can cause hepatorenal failure. The rash is macular and there is an eshcar ("tache noire") at the entry site in the majority of cases. Mediterranean tick typhus ("fièvre bouttonneuse") is the type most likely to be imported into the UK but only a handful of cases are reported annually.

Scrub typhus is clinically similar to Old World tick typhus, with an eschar in up to $80 \%$

\section{Learning points}

- The types of typhus fever are:

(A) Epidemic louse borne: $R$ prowazekii

(B) Endemic flea borne: $R$ typhi/R mooseri

(C) Tick borne "spotted fever": $R$ rickettsii, $R$ conorii, etc

(D) Mite borne scrub typhus: O tsutsugamushi

- The typhus fevers all present with headache and a macular rash. A clinical diagnosis is often made, and a specific therapeutic trial undertaken, before serological confirmation is received.

- Careful examination of the patient for the entry site eschar (insect bite) is helpful in the diagnosis of scrub and tick typhus, the commonest types in travellers.

- In Thailand there is evidence of resistance of scrub typhus to chloramphenicol and tetracycline. Possible alternative treatments include rifampicin and ciprofloxacin.

- Louse borne typhus epidemics are a risk in all refugee camps, especially in the cooler mountainous regions of tropical countries, but also in European settings.

- Foci of scrub typhus in jungle areas of South East Asia are maintained by vertical transmission in the mite vectors without the need for mammal hosts.

of cases, typically in the groin or axilla (see fig 1 ), and the potential for an acute brain syndrome which can progress to coma and, rarely, death. Occasional imported cases are reported in the UK.

The clinical severity of epidemic typhus is largely determined by the nutritional state of the population infected and in the most extreme situations of malnutrition, such as in the concentration camps at Bergen-Belsen in 1945, a mortality of $50 \%$ and upwards may occur. After an incubation period of about 14 days, there is a sudden onset of high prostrating fever with severe headache, limb pains, and vomiting. The face is suffused and the victim appears vacant and semimute, with epistaxis and a dry cough. The classical rash appears on about the third day on the trunk (see fig 2) and proximal limbs, consisting of irregular pink macules, which darken and coalesce but are not haemorrhagic. Constipation is usual and paralytic ileus may ensue. Splenomegaly and generalised lymphadenopathy are common. In severe cases a meningoencephalitic syndrome develops, with meningism, tinnitus and hyperacusis, followed by deafness, dysphoria, agitation, and coma. Survivors may have hemiparesis, acute transverse myelitis, or peripheral neuropathy. Other possible complications include: (1) secondary bacterial infection, leading to bronchopneumonia, parotitis, or otitis media; (2) myocarditis, presenting as low output cardiac failure or sudden collapse, typically when the generalised febrile illness is recovering; and (3) peripheral gangrene or venous thromboembolism. 


\section{Diagnosis}

Because of the lack of clues from routine laboratory investigations, which are usually normal, and the facts that rickettsiae are dangerous pathogens and specific serological tests are only available from a few specialised laboratories, often in retrospect, it is advisable to make a clinical diagnosis of the typhus fevers based on a detailed travel and occupational history and the physical signs. In these circumstances a therapeutic trial of specific treatment is justified, as rapid defervescence is strongly suggestive of a correct diagnosis.

The differential clinical diagnosis includes typhoid (enteric) fever, viral haemorrhagic fever, meningococcal infection, louse borne relapsing fever, leptospirosis and, as for all fevers acquired in the tropics, malaria, which should always be excluded by the examination of blood films.

Specific diagnosis can be made by:

(1) The Weil-Felix test, involving the demonstration of heterophile antibodies to strains of Proteus mirabilis (OX-19, OK-2, OX-K); this test is, however, negative in up to $50 \%$ of cases.

(2) Group specific microagglutination test or species specific immunofluorescent antibody test (IFAT) or enzyme linked immunoabsorbent assay (ELISA).

An indirect immunoperoxidase version of the IFAT is highly sensitive, ${ }^{2}$ and the ELISA can be applied to filter paper for field use.

(3) Polymerase chain reaction techniques. ${ }^{17}$

\section{Management}

Good general care of the patient is essential, emphasising fluid balance, oral toilet, analgesia, mild sedation if needed, and appropriate antibiotics for secondary infections. Acute renal failure needs appropriate management and modification of the antibiotic regimen.

Specific chemotherapy with chloramphenicol, first used in scrub typhus in $1947,{ }^{18}$ is with $500 \mathrm{mg}$ six hourly orally or intravenously for seven to 10 days in adults, or $12.5 \mathrm{mg} / \mathrm{kg}$ six hourly for children. If tetracycline is preferred, the same dosages are used. In all mild to moderate cases, however, the treatment of choice is oral doxycycline $200 \mathrm{mg}$ for adults, $100 \mathrm{mg}$ for children, in a single dose, with one further dose in five to seven days if relapse of fever occurs.

There are now reliable reports ${ }^{4}$ of partial resistance to chloramphenicol and tetracycline, for $O$ tsutsagamush $i$ only, in northern Thailand. Both are bacteriostatic agents so that in cases acquired there it may be wise to treat scrub typhus $a b$ initio with rifampicin or a quinolone antibiotic, for example ciprofloxacin.

\section{Prevention and control}

In epidemics of louse borne typhus it is essential to treat all cases with doxycycline, and indeed to consider mass prophylaxis with this agent in the whole population at risk and for carers and medical workers on a weekly basis. All those at risk should initially be issued with clean clothes, which are dusted with powder containing $1 \%$ malathion or $1 \%$ permethrin. Again, this should include medical attendants, since history shows that they are at high risk.
Multiple choice questions (answers at end of paper)

1. Scrub typhus in South East Asia is transmitted by:

(A) Ticks from wild animals

(B) Rat fleas

(C) Larval mites on long grass

(D) Head lice

(E) Sandflies

2. Epidemic louse borne typhus:

(A) Typically has a haemorrhagic rash and evidence of diffuse intravascular coagulation

(B) In tropical countries occurs most in seaside areas of high temperature and humidity

(C) In undernourished refugees is likely to carry a high mortality if untreated

(D) Can lead to peripheral gangrene and loss of digits in severe cases

(E) Should be controlled by the widespread prescription of ciprofloxacin in the community at risk

3. Possible treatments for the typhus fevers include:

(A) Doxycycline

(B) Ciprofloxacin

(C) Chloramphenicol

(D) Erythromycin

(E) Coamoxiclav

4. Recognised effective methods of prevention of scrub and tick typhus include:

(A) The wearing of clothing impregnated with DEET

(B) Specific vaccination

(C) The use of bed nets impregnated with permethrin

(D) The wearing of stout boots, gaiters, and long trousers

(E) Daily oral doxycycline $100 \mathrm{mg}$

5. Areas of risk of tick borne typhus include:

(A) Queensland, Australia

(B) Rocky Mountains, USA

(C) Game parks in Zimbabwe

(D) Mediterranean coast of France

(E) Scottish Highlands

Endemic flea borne typhus can be controlled by antirodent measures, and individual use of insect repellent creams.

Measures to reduce the risk of tick typhus include the control of dog ticks, "buddy" inspection for ticks after visits to game parks or forests, insect repellents, and the impregnation of clothing with DEET (diethyl toluamide) or permethrin.

Scrub typhus control depends on the clearing of secondary jungle grass within and near villages, and, in travellers, the use of jungle boots and gaiters with long trousers, permethrin or DEET impregnation of clothing, and consideration of the use of prophylactic oral doxycycline $200 \mathrm{mg}$ weekly in those who have to travel through areas of known high risk, for example soldiers, policemen, plantation workers. 
1 Cowan G. Rickettsial diseases. In: Cook GC, ed. Manson's tropical diseases. 20th Ed. London: Saunders, 1996.

Roult D. Ndihokubwayo JB, Tissot-Dupont $\mathrm{H}$, et al. Outbreak of epidemic typhus associated with trench fever in Burundi. Lancet 1998;352:353-8.

3 Luxemburger C, Rijal J, Nosten F. Health care in refugee camps. Trans $R$ Soc Trop Med Hyg 1998;92:129-30.

4 Watt G, Chouriyagune C, Ruangweeraynd R, et al. Scrub typhus infections poorly responsive to antibiotics in northern Thailand. Lancet 1996;348:86-9.

5 Hackstadt T. The biology of rickettsiae. Infect Agents Dis 1996;5:127-43.

6 Zinsser H. Varieties of typhus vaccine and the epidemiology of the American form of European typhus fever (Brill's disease). American fournal of Hygiene 1934;20:513-32.

7 Anderson SGE, Zomorodipour A, Anderson JO, et al. The genome sequence of Rickettsia prowazekii and the origin of mitochondria. Nature 1998;396:133-40.

8 Pether JVS, Jones W, Lloyd G, et al. Fatal murine typhus from S S, Jones W, Lloyd G, et

from Spain. Lancet 1994;344:897-8.
Sanchez JL, Candler WH, Fishbein DB, et al. A cluster of tick-borne infections; association with military training and asymptomatic infections due to Rickettsia rickettsii. Trans $R$ Soc Trop Med Hyg 1992; 86:321-5.

10 Senneville E, Ajana F, Lecoq P, et al. Rickettsia conorii, isolated from ticks introduced to northern France by a dog. Lancet 1991;337:676.

11 Kelly PJ, Beati L, Matthewman LA, et al. A new pathogenic spotted fever group rickettsia from Africa. 7 Trop Med Hyg 1994;97:129-37.
12 Sirisanthana T, Pinyoporupanit V, Sirisanthana V, et al. First cases of spotted fever group rickettsiosis in Thailand. $A m \mathcal{F}$ Trop Med Hyg 1994;50:682-6.

13 Baird RW, Lloyd M, Stenos J, et al. Characterization and comparison of Australian spotted fever group rickettsiae. 7 Clin Microbiol 1992;30:2896-902.

14 Tamura A, Ohashi N. Urakami $\mathrm{H}$, et al. Classification of Rickettsia tsutsugamushi in a new genus Orientia gen nov as Orientia tsutsugamushi comb.nov. Int f Syst Microbiol 1995; 45:589-91.

15 Wohlbach SB. The aetiology and pathology of typhus. Cambridge, MA: Harvard University Press, 1922

16 Brown GW, Shirai A, Jegathesan M. Febrile illness in Malaysia - an analysis of 1629 hospitalised patients. Am f Trop Med Hyg 1984;33:31 1-15.

17 Sugita Y, Nagatani T, Okuda K, et al. Diagnosis of typhus infection with Rickettsia tsutsugamushi by polymerase chain reaction. $f$ Med Micriobiol 1992;37:357-60.

18 Smadel JE, Woodward TE, Ley H. Chloramphenicol (chloromycetin) in the treatment of tsutsugamushi disease (scrub typhus). 7 Clin Invest 1949;28:1196-215.

\section{Answers: true (T)/false (F)}

1. (A) F, (B) F, (C) T, (D) F, (E) F; 2. (A) F, (B) F, (C) T, (D)

$\mathrm{T}$, (E) F; 3. (A) T, (B) T, (C) T, (D) F, (E) F; 4. (A) T, (B) F,

(C) F, (D) T, (E) F; 5. (A) T, (B) F, (C) T, (D) T, (E) F 